\title{
Particle size distributions from spectral turbidity: a singular-system analysis
}

\author{
M Bertero†, C De Molł and E R Pike§ \\ + Dipartimento di Fisica dell'Università and Istituto Nazionale di Fisica Nucleare, Via \\ Dodecaneso, 33, I-16146 Genova, Italy \\ \$Département de Mathématique, Université Libre de Bruxelles, Campus Plaine C.P.217, \\ Boulevard du Triomphe, B-1050 Bruxelles, Belgium \\ $\S$ Royal Signals and Radar Establishment, St Andrews Road, Great Malvern, \\ Worcestershire WR14 3PS, UK
}

Received 15 November 1985, in final form 10 December 1985

\begin{abstract}
The problem of the inversion of spectral turbidity measurements to obtain distributions of droplet size in polydisperse aerosols is considered by introducing a recently developed generalised theory of information based on singular-system analysis. In this work we limit ourselves to the anomalous scattering approximation but give for this case an extensive treatment, with examples, of the reconstruction of the distribution as a continuous function in both weighted and unweighted $L^{2}$ spaces from finite sampled data sets. The methods are entirely parallel to those already used successfully in the related problems of particle size distribution by photon correlation spectroscopy and by Fraunhofer diffraction.
\end{abstract}

\section{Introduction}

In a number of recent works [1-8] a generalised information theory has been developed which extends the classical theory of information due to Shannon and others in a natural way and allows the same basic concepts of extraction of signals from noise up to given resolution limits to be used in more general problems such as the one described in this paper. The classical theory makes use of the properties of spectral representations of selfadjoint operators, while the extension to the case of more general operators requires the introduction of their 'singular systems' which consist of a triplet of (i) a basis set in an object space, (ii) a basis set in a 'data space' related reciprocally to (i) by the operator and its adjoint and (iii) a set of scalar singular values which are the analogues of the eigenvalues of the conventional theory. This gives a framework for a discussion of inversion along similar lines to those of the classical theory in these more general cases where the classical theory does not apply.

The common feature of the type of problem under discussion is that they can all be described by an underlying Fredholm equation of the first kind:

$$
g(x)=\int K(x, y) f(y) \mathrm{d} y .
$$

For particle size distribution measurements by photon correlation spectroscopy [9] $K(x, y)$ is $\exp (-x y)$ and the problem of inversion is that of the Laplace transform. For Fraunhofer diffraction $[8,10] K(x, y)$ is $J_{1}^{2}(x y) /(x y)^{2}$ and there are, of course, numerous other problems with their own specific forms of kernel. 
The kernel we consider in this paper relates the data obtained in a spectral turbidity measurement to the particle size distribution in the anomalous scattering approximation [11]. We use this approximation to illustrate the method and to take advantage of the analytic simplicity of some of the results, but the same considerations may be applied to the Mie scattering kernel for wider experimental application at the cost of somewhat more lengthy computations.

The method, in its present form, applies strictly to the case of additive noise. Although correlated additive noise can be treated, the most straightforward calculations result if it can be arranged that the data are expressed in such a way that the experimental noise is white. The first step required, therefore, is to cast the Fredholm equation into a form in which the noise on the data points is as white as possible. The scaling factors required to achieve this may be different in various experimental circumstances. For example, in the case of the Fraunhofer scattering experiment, photodiode detectors with noise proportional to detector area will require different kernels according to the disposition and relative sizes of the detectors at different scattering angles.

For the purposes of the present paper we shall consider an experimental arrangement in which we have a photoconductive or photovoltaic detector with energy response, $j(k)$, as a function of wavenumber, $k$, related to the photon number flux by $j(k)=\eta k n(k)$, where $\eta$ is the quantum efficiency, and with a constant, additive electrical noise on $j(k) / k$. Under these circumstances the unknown function of the particle size which will appear in the inversion will turn out to be, conveniently, the volume distribution. We again note that there is no degree of freedom in the power of the radius to be used; this is entirely determined by the experimental noise sources.

The integral equation conventionally used for this problem applies to the case of dilute scatterers where the extinction, $\tau$, given by

$$
I / I_{0}=\exp (-\tau / S)
$$

where $S$ is the effective beam area, is also found from the expansion

$$
\tau(k)=S\left(1-I(k) / I_{0}(k)\right) \simeq S\left(1-j(k) / j_{0}(k)\right)
$$

or, in terms of the photon flux,

$$
g(k)=\frac{\tau(k)}{k}=\frac{S}{k}-\frac{S \eta n(k)}{j_{0}(k)}+\text { white noise. }
$$

We assume that the source properties are known without error. For this scaled data function, equation (1.1) applies, changing $x$ to $k$ and $y$ to particle radius, $r$, and using $\rho$ for the product $k r$, with the following identifications:

$$
\begin{aligned}
K(\rho) & =3\left(2+\frac{4}{\rho^{2}}-\frac{4 \sin \rho}{\rho}-\frac{4 \cos \rho}{\rho^{2}}\right) / 4 \rho \\
& =3(\pi / 2)^{1 / 2} \rho^{-3 / 2} \boldsymbol{H}_{3 / 2}(\rho)
\end{aligned}
$$

where $\boldsymbol{H}_{\nu}(\rho)$ is the Struve function of order $\nu$ and

$$
f(r)=\frac{4}{3} \pi r^{3} N(r)
$$

where $N(r)$ is the particle radius distribution. 
We might also note at this point that there is extensive previous literature on this problem, including various exact inversion formulae, some of which are even not illconditioned. From the practical point of view, however, unfortunately the majority of this work has not enjoyed any significant success. In particular, exact inversion formulae, dating back to Shifrin and Perelman [12] require a priori knowledge of parameters of the distribution which are not readily available in most cases. In spite, therefore, of this volume of work, apart from a few isolated cases [13] there is still a strong requirement for illustrated practical examples of successful inversions.

To provide a basis for comparison for the results to be presented later we first consider the hypothetical case where the data are known over the complete semi-axis $0<k<\infty$ and we reconstruct solutions over the entire semi-axis of $r, 0<r<\infty$. We thus have the problem of the inversion of the integral operator $K$, defined by

$$
(K f)(k)=\int_{0}^{\infty} K(k r) f(r) \mathrm{d} r .
$$

This operator is bounded and self-adjoint in $L^{2}(0, \infty)$ and its spectral representation may be obtained using the results of McWhirter and Pike [14].

In order to apply these latter results we need the Mellin transform of $K(\rho)$ which may be found using the well known formula

$$
\int_{0}^{\infty} t^{\mu-\nu-1} \boldsymbol{H}_{\nu}(t) \mathrm{d} t=\frac{2^{\mu-\nu-1} \Gamma(\mu / 2) \tan (\pi \mu / 2)}{\Gamma(\nu+1-\mu / 2)}
$$

where

$$
|\operatorname{Re}(\mu)|<1 \text { and } \operatorname{Re}(\nu)>\operatorname{Re}(\mu)-\frac{3}{2}
$$

Putting $\nu=\frac{3}{2}$ and using the notation of McWhirter and Pike this transform is

$$
\tilde{K}(\mu)=3 \sqrt{\pi / 2} \frac{2^{\mu-5 / 2} \Gamma(\mu / 2) \tan (\pi \mu / 2)}{\Gamma\left(\frac{5}{2}-\mu / 2\right)} .
$$

Again from McWhirter and Pike the eigenvalues are given by

$$
\lambda_{\omega}^{ \pm}=\left|\tilde{K}\left(\frac{1}{2}+i \omega\right)\right| \text {. }
$$

After some manipulations we find

$$
\lambda_{\omega}^{ \pm}=\frac{(72 \pi)^{1 / 2}}{\left[\left(25+4 \omega^{2}\right)\left(1+4 \omega^{2}\right)\right]^{1 / 2}} .
$$

This spectrum is shown in figure 1 , together with resolution limits, $\delta$, defined by $\delta=$ $\exp \left(\pi / \omega_{\max }\right)$ as in the work, for example, of Bertero and Pike on the Fraunhofer diffraction problem [10]. $\omega_{\max }$ is an upper 'frequency' cut-off determined by the noise level and $\delta$ is a dilation factor which is the ratio of the closest distances apart for which independent estimates can be made of values of the reconstructed solution.

In $\S 2$ we calculate singular systems for distributions with bounded support, the bounds supposed known a priori, with various types of hypothetical and achievable data sets. These include a knowledge of the data over the full real semi-axis of $k$, the results of which may be compared with others obtained using truncated and sampled data with different spacings and ranges.

In the following section we extend the analysis to the case of weighted space reconstructions, where use of a 'weighting function' allows a priori probabilistic or 


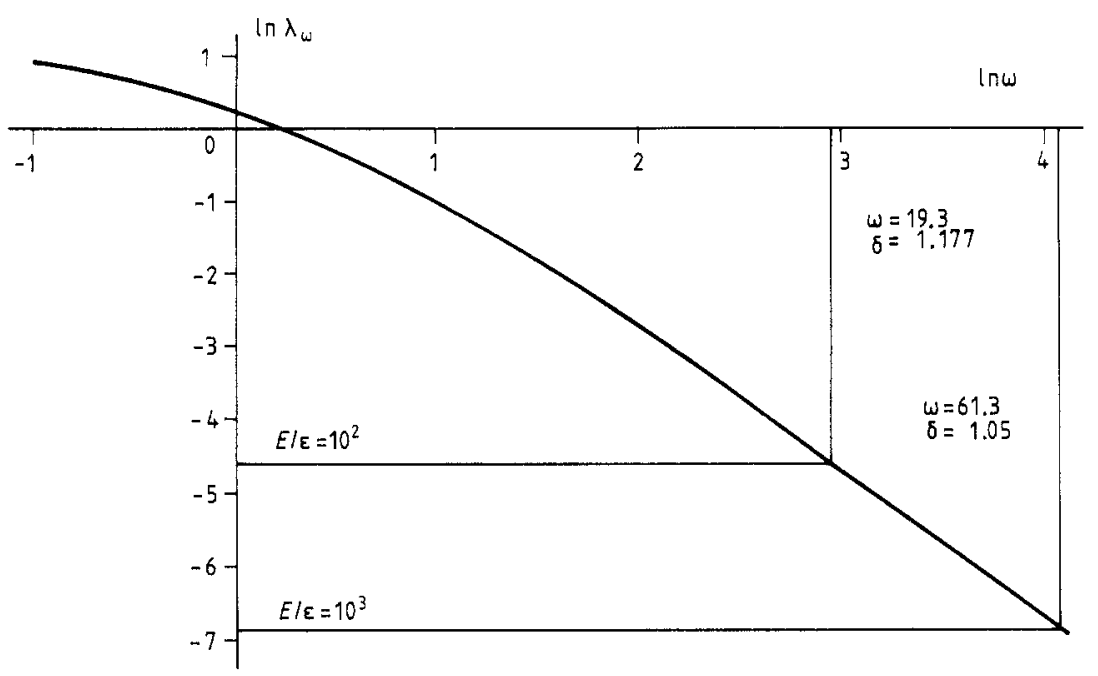

Figure 1. The spectrum for full continuous data as given by (1.12), with the corresponding resolution limits $\delta$ for two values of the signal-to-noise ratio $E / \varepsilon$.

approximate knowledge of the form of the distribution to be incorporated and hence improve the resolution in reconstruction [15-18]. The method also allows the behaviour of the reconstruction at the edges of its support to be controlled and avoid undesirable 'ringing' effects which may occur when a sharp cut-off is used. The paper is concluded with a section in which reconstructions are calculated from various sets of simulated data.

\section{The case of a distribution with bounded support}

In this section we investigate the case where the unknown particle distribution is assumed to be supported in the known bounded interval $\left[r_{1}, r_{2}\right]$ with $0<r_{1}<r_{2}<\infty$. The purpose is to try to compensate the loss of information due to limited data by introducing a priori knowledge of the support of the unknown function.

\subsection{Continuous data}

Assume that the function $g(k)$ is measured in the interval $\left[k_{1}, k_{2}\right]$ with $k_{1} \geqslant 0, k_{2} \leqslant \infty$ (the results given in this section hold also in the case $k_{1}=0$ and/or $\left.k_{2}=\infty\right)$. Then the operator (1.7) becomes an operator from $L^{2}\left(r_{1}, r_{2}\right)$ into $L^{2}\left(k_{1}, k_{2}\right)$, given by

$$
(K f)(k)=\int_{r_{1}}^{r_{2}} K(k r) f(r) \mathrm{d} r \quad k_{1} \leqslant k \leqslant k_{2} .
$$

It is convenient to introduce dimensionless variables by putting

$$
y=r / r_{1} \quad x=r_{1} k \quad \gamma=r_{2} / r_{1}
$$

so that, by an appropriate scaling of the solution and of the data, we get

$$
(K f)(x)=\int_{1}^{\gamma} K(x y) f(y) \mathrm{d} y \quad x_{1} \leqslant x \leqslant x_{2}
$$


where $x_{i}=r_{1} k_{i}(i=1,2)$. The operator $K: L^{2}(1, \gamma) \rightarrow L^{2}\left(x_{1}, x_{2}\right)$ is compact. We denote its singular system by $\left\{\alpha_{k} ; u_{k}, v_{k}\right\}_{k=0}^{\infty}$, the set of the solutions of the coupled equations:

$$
K^{*} v_{k}=\alpha_{k} u_{k} \quad K u_{k}=\alpha_{k} v_{k}
$$

where $K^{*}: L^{2}\left(x_{1}, x_{2}\right) \rightarrow L^{2}(1, \gamma)$ is the adjoint operator given by

$$
\left(K^{*} g\right)(y)=\int_{x_{1}}^{x_{2}} K(y x) g(x) \mathrm{d} x \quad 1 \leqslant y \leqslant \gamma .
$$

We notice that the operator $K^{*} K$ has a kernel given by

$$
T\left(y, y^{\prime}\right)=\int_{x_{1}}^{x_{2}} K(y x) K\left(y^{\prime} x\right) \mathrm{d} x
$$

(with $y, y^{\prime} \in[1, \gamma]$ ) which is an analytic function of $y, y^{\prime}$ in a neighbourhood of the square $[1, \gamma] \times[1, \gamma]$. Indeed, from the inequality

$$
|K(\rho)| \leqslant a \rho\left(b+\rho^{2}\right)^{-1}
$$

which can be easily devised by looking at the behaviour of $K(\rho)$ for $\rho \rightarrow 0$ and $\rho \rightarrow \infty$, it follows that the integral in equation (2.6) is absolutely and uniformly convergent with respect to $y, y^{\prime}$. Then, as in [8], the following results can be easily proved.

(i) The operator $K$ is invertible, i.e. the equation $K f=0$ has only the trivial solution $f=0$.

(ii) The singular values $\alpha_{k}$ tend to zero exponentially fast when $k \rightarrow \infty$, since the $\alpha_{k}^{2}$ are the eigenvalues of the operator $K^{*} K$ whose kernel is analytic [19].

(iii) The first singular value $\alpha_{0}$ is bounded by

$$
\alpha_{0}<\lambda_{0}^{+}
$$

where $\lambda_{0}^{+}=\sqrt{72 \pi} / 5=3.00795$.

(iv) If we denote by $J$ the interval $\left[x_{1}, x_{2}\right]$ and by $\alpha_{k}(\gamma, J)$ the singular values, in order to make explicit their dependence on the support of the solution and of the data, then

$$
\alpha_{k}(\gamma, J) \leqslant \alpha_{k}\left(\gamma^{\prime}, J^{\prime}\right)
$$

if $\gamma\left\langle\gamma^{\prime}, J \subset J^{\prime}\right.$; i.e. the singular values are increasing functions of the support of the data and of the solution. Furthermore, when $\gamma$ is larger and the data support tends to cover $(0,+\infty)$ the $\alpha_{k}(\gamma, J)$ are given approximately by

$$
\alpha_{k}(\gamma, J) \simeq\left(\frac{72 \pi}{\left(25+4 \omega_{k}^{2}\right)\left(1+4 \omega_{k}^{2}\right)}\right)^{1 / 2}
$$

where

$$
\omega_{k}=\pi k / \ln \gamma
$$

(v) When $J$ and $\gamma$ are large, the singular functions $u_{k}$ satisfy approximately the 'symmetry properties':

$$
u_{k}(y)=(-1)^{k} \frac{\sqrt{\gamma}}{y} u_{k}\left(\frac{\gamma}{y}\right)
$$

and therefore the 'odd' singular functions have a zero at $y=\sqrt{\gamma}$. 


\subsection{Discrete data}

When the data function is measured only on a finite set of points $\xi_{1}, \xi_{2}, \ldots, \xi_{N}$ then, as in similar problems $[7,8]$, one has to invert a linear operator transforming a function $f$ into a finite-dimensional vector:

$$
\left(K_{N} f\right)\left(\xi_{n}\right)=\int_{1}^{\gamma} K\left(\xi_{n} y\right) f(y) \mathrm{d} y \quad n=1, \ldots, N .
$$

If the space of data vectors is equipped with a scalar product having the form

$$
\left(g_{1}, g_{2}\right)=\sum_{n=1}^{N} w_{n} g_{1}\left(\xi_{n}\right) g_{2}\left(\xi_{n}\right)
$$

then, the adjoint operator $K_{N}^{*}$ which transforms a data vector into a function, is given by

$$
\left(K_{N}^{*} g\right)(y)=\sum_{n=1}^{N} w_{n} K\left(\xi_{n} y\right) g\left(\xi_{n}\right) .
$$

We denote by $\left\{\alpha_{N, k} ; u_{N, k}, v_{N, k}\right\}_{k=0}^{\infty}$ the singular system of the operator $K_{N}$.

Notice that, since the functions $K\left(\xi_{n} y\right)$ are linearly independent, the operator $K_{N}^{*}$ is injective and therefore the operator $K_{N}$ has exactly $N$ positive singular values $\alpha_{N, k}$. These are also the square roots of the eigenvalues of the finite-rank integral operator $K_{N}^{*} K_{N}$, whose kernel is given by

$$
T_{N}\left(y, y^{\prime}\right)=\sum_{n=1}^{N} w_{n} K\left(\xi_{n} y\right) K\left(\xi_{n} y^{\prime}\right) .
$$

This kernel appears as an approximation, given by some quadrature formula, of the kernel (2.6).

As in our previous work on Laplace transform inversion [7] and Fraunhofer diffraction [8] we consider two data-point distributions: the first is a set of equidistant points:

$$
\xi_{n}=x_{1}+d(n-1) \quad n=1, \ldots, N
$$

where $d$, the distance between adjacent points, is such that $\xi_{N}=x_{1}+(N-1) d=x_{2}$; the second is a set of points following a geometric progression:

$$
\xi_{n}=x_{1} \Delta^{n-1} \quad n=1, \ldots, N
$$

where $\Delta$ (dilation factor) is such that $\xi_{N}=x_{1} \Delta^{N-1}=x_{2}$.

In the case of equidistant points we take constant weights:

$$
w_{n}=d
$$

while in the case of points forming a geometric progression we take [7]

$$
w_{n}=(\ln \Delta) \xi_{n} .
$$

As in $[7,8]$ it is easy to prove that, when $N$ tends to infinity and the distance between adjacent points tends to zero (in the case $(2.18)$ that means $\Delta \rightarrow 1$ ) in such a way that $\xi_{N} \rightarrow \infty$, then the kernel (2.16) converges uniformly to the kernel (2.6) and therefore from some perturbative lemmas for compact operators [20] it follows that the singular values $\alpha_{N, k}$ converge to $\alpha_{k}$ and the singular functions $u_{N, k}$ converge (in the norm of $L^{2}$ ) to $u_{k}$. 


\section{Inversion in weighted spaces}

The disadvantage of the inversions based on the assumption of a bounded support of the solution is that the singular functions are large at the edges of the support. This difficulty can be avoided by introducing a suitable weighted space $[8,16]$, i.e., by assuming that $f$ satisfies the condition

$$
\int_{0}^{\infty} \frac{f^{2}(r)}{P^{2}(r)} \mathrm{d} r<\infty
$$

where $P(r)$ is a given 'profile function' expressing some a priori knowledge of the shape of $f(r)$.

If we put

$$
f(r)=P(r) \varphi(r)
$$

then the problem of the inversion of the operator (1.7) on the space of the functions satisfying condition ( 3.1$)$ is equivalent to the problem of the inversion in $L^{2}(0,+\infty)$ of the operator

$$
(K \varphi)(k)=\int_{0}^{\infty} K(k \rho) P(\rho) \varphi(\rho) \mathrm{d} \rho \quad 0<k<\infty .
$$

By using the formula [21]

$$
\int_{0}^{\infty} \boldsymbol{H}_{\nu}(t) \boldsymbol{H}_{\mu}(t) t^{-(\mu+\nu)} \mathrm{d} t=\frac{\sqrt{\pi} \Gamma(\mu+\nu) 2^{-(\mu+\nu)}}{\Gamma\left(\mu+\nu+\frac{1}{2}\right) \Gamma\left(\mu+\frac{1}{2}\right) \Gamma\left(\nu+\frac{1}{2}\right)}
$$

$(\operatorname{Re}(\mu+\nu)>0)$ it is easy to see that

$$
\operatorname{Tr}\left(K^{*} K\right)=\frac{9 \pi}{15} \int_{0}^{\infty} \frac{P^{2}(y)}{y} \mathrm{~d} y .
$$

Therefore, if the profile $P(y)$ is such that the integral on the right-hand side of equation (3.5) converges, the operator $K^{*} K$ is of the trace class and hence $K$ is compact.

The case of a bounded support of the data and of discrete data can be treated as in $[7,8]$. In these papers we mainly considered the case where $P(y)$ is a gamma distribution. By an appropriate scaling of the variables it is always possible to ensure that the first moment of $P(y)$ is equal to one, so that

$$
P(y)=\frac{\beta^{\beta} y^{\beta-1} \mathrm{e}^{-\beta y}}{\Gamma(\beta)} \quad \beta>1 .
$$

In this case the integral (3.5) can be computed and it gives an upper bound to the first singular value.

\section{Numerical results and simulations}

Using the method described in $[7,8]$ we have performed numerical computations of the singular system of the operator $K_{N}$ for different data-point distributions and choices of the profile function $P(r)$. Because of noise, only the largest singular values are relevant for practical inversions so that we computed only the singular values $\alpha_{N, k}$ larger than $10^{-3}$ 

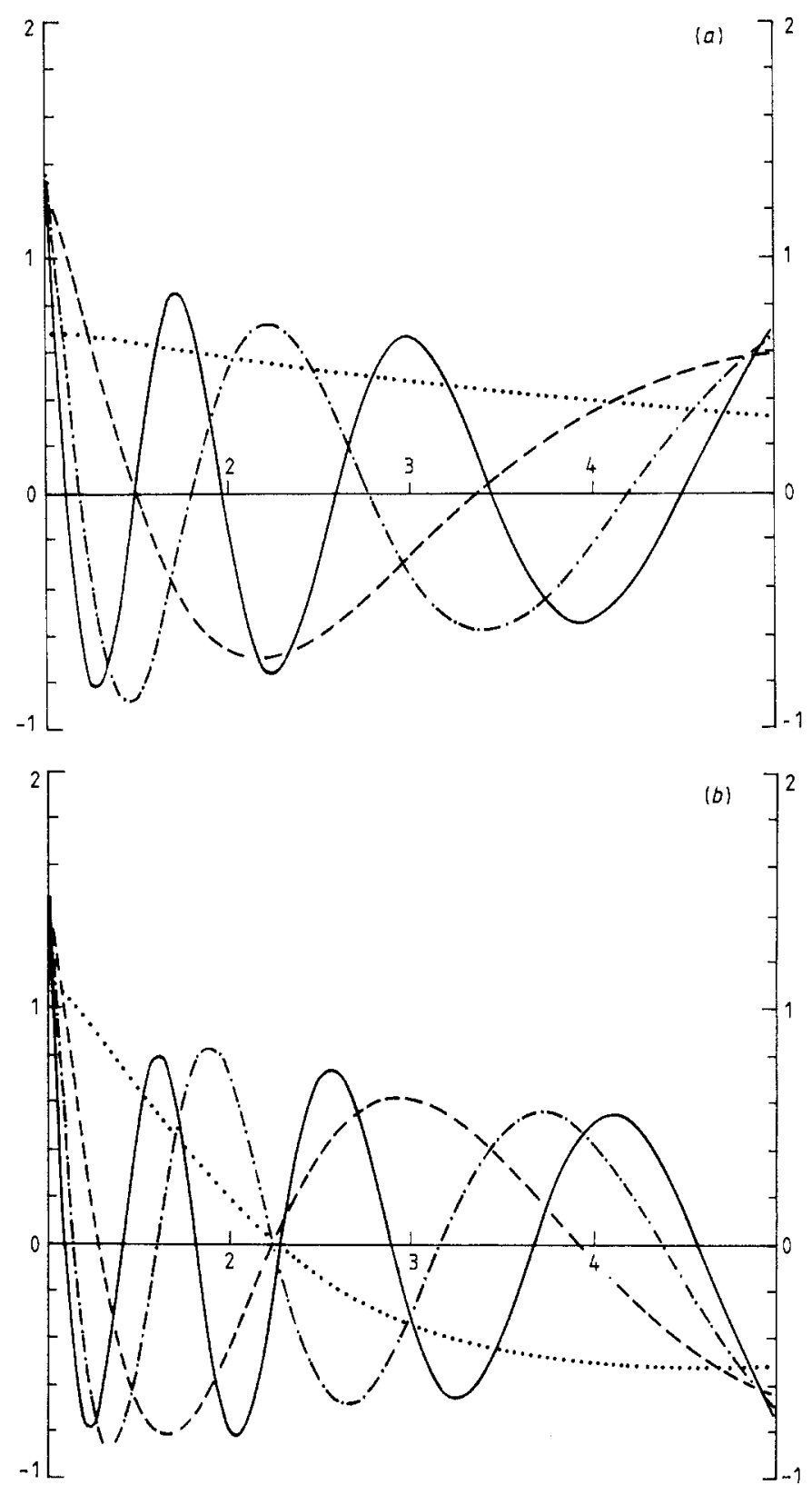

Figure 2. Singular functions in the case of an unknown function with bounded support $[1, \gamma]$, $\gamma=5$, and of 31 equidistant data points on $\left[x_{1}, x_{2}\right], x_{1}=0.001, x_{2}=10$. (a) Singular functions with $k$ even: $u_{0}$ (dotted curve), $u_{2}$ (broken curve), $u_{4}$ (chain curve) and $u_{6}$ (full curve); (b) singular functions with $k$ odd: $u_{1}$ (dotted curve), $u_{3}$ (broken curve), $u_{5}$ (chain curve) and $u_{7}$ (full curve).

and the corresponding singular functions $u_{N, k}$. We report here two typical examples of singular functions.

In figure 2 we give the eight first singular functions of the operator $K_{N}$, given by (2.13), in the unweighted case, for $\gamma=5$ and for a set of $N=31$ equidistant data points (see (2.17)) 

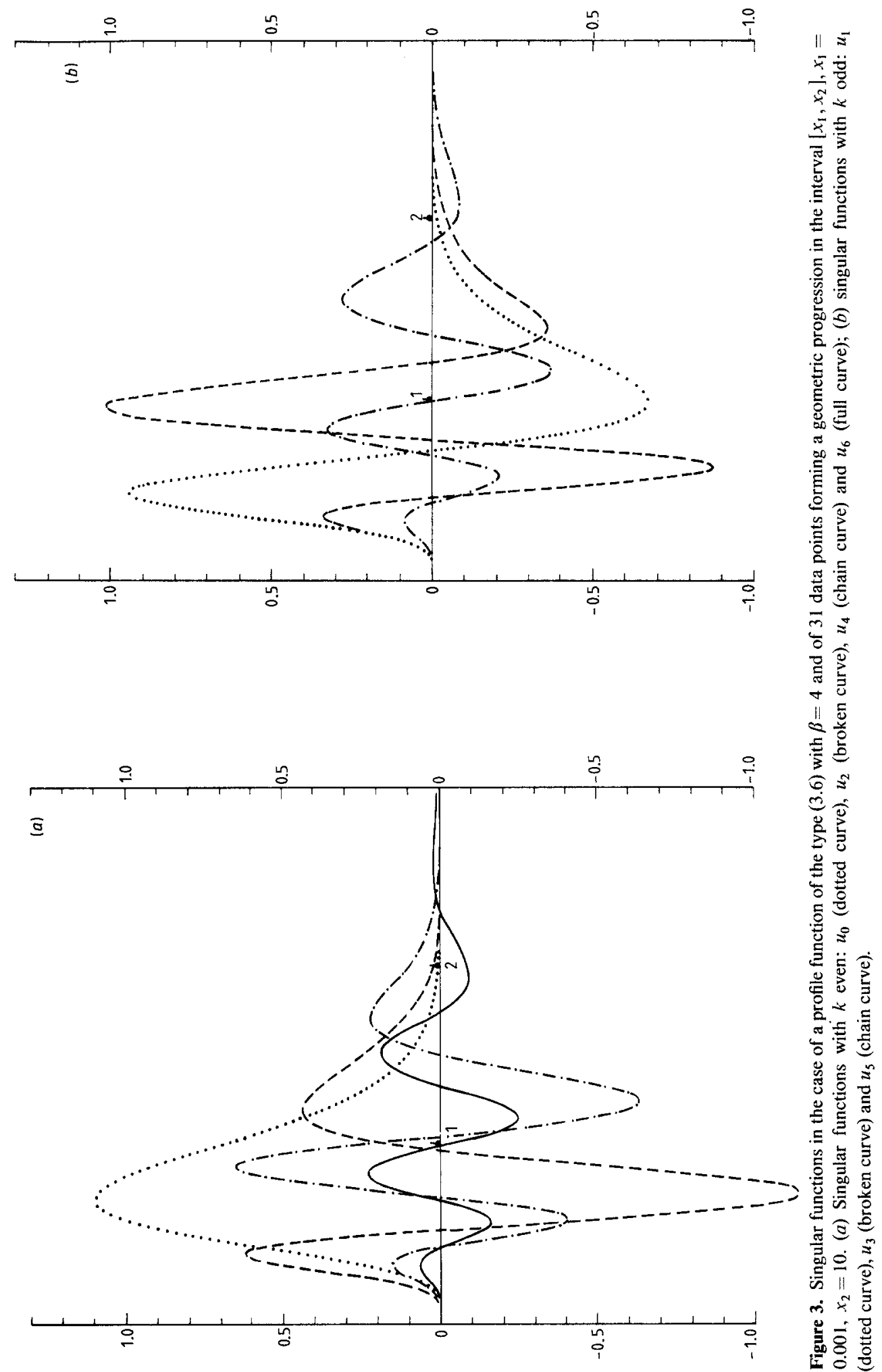
in the interval $\left[x_{1}, x_{2}\right]$, with $x_{1}=0.001, x_{2}=10$ and equidistance $d=0.3333$. The corresponding singular values are given by

$$
\begin{array}{ll}
\alpha_{N, 0}=1.5822 & \alpha_{N, 4}=0.6635 \times 10^{-1} \\
\alpha_{N, 1}=0.5308 & \alpha_{N, 5}=0.4272 \times 10^{-1} \\
\alpha_{N, 2}=0.2224 & \alpha_{N, 6}=0.2934 \times 10^{-1} \\
\alpha_{N, 3}=0.1336 & \alpha_{N, 7}=0.2103 \times 10^{-1} .
\end{array}
$$

In figure 3 we give the first seven singular functions $u_{N, k}$ corresponding to the choice (3.6) for the profile function, with $\beta=4$. The data set was a set of 31 points forming a geometric progression as given by $(2.18)$ with $x_{1}=0.001, x_{2}=10$, and hence $\Delta=1.359356$. The corresponding singular values are given by

$$
\begin{array}{ll}
\alpha_{N, 0}=1.0811 & \alpha_{N, 4}=0.2816 \times 10^{-1} \\
\alpha_{N, 1}=0.3105 & \alpha_{N, 5}=0.7275 \times 10^{-2} \\
\alpha_{N, 2}=0.1393 & \alpha_{N, 6}=0.1036 \times 10^{-2} \\
\alpha_{N, 3}=0.6827 \times 10^{-1} &
\end{array}
$$

and the succeeding singular values are smaller than $10^{-3}$.

We have also performed reconstructions of distributions $f(r)$ from simulated data. As in $[7,8]$ the reconstructions have been obtained by expanding the solution $f(r)$ in the

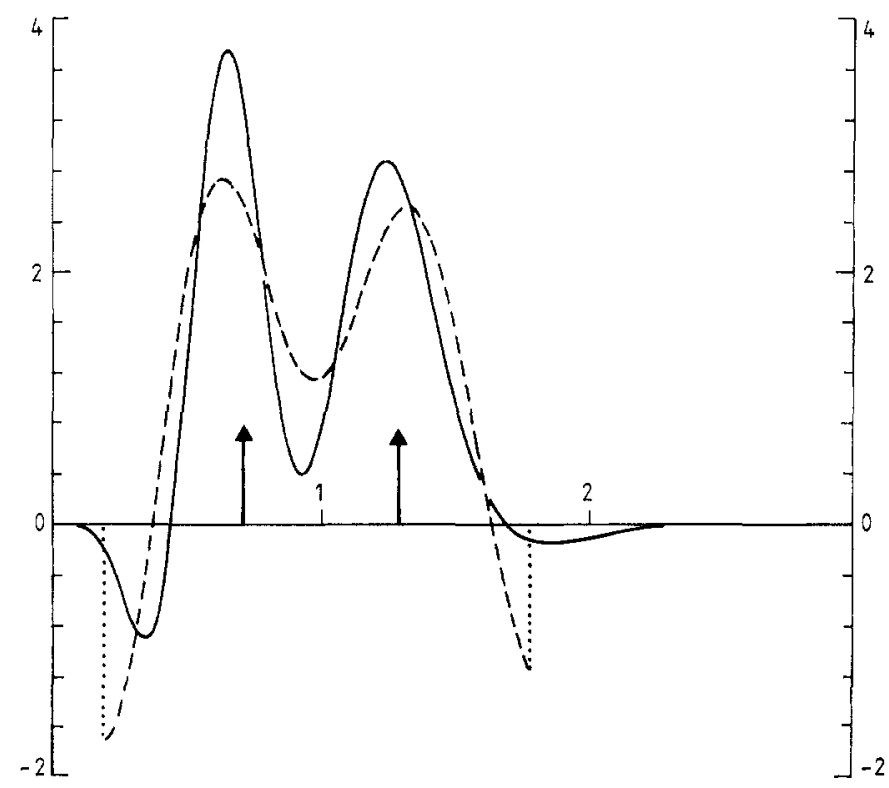

Figure 4. Reconstruction of a sum of two delta functions centred at 0.725 and 1.275 , indicated by the arrows. The singular functions of figure 3 were used up to $k=4$, i.e. assuming a signal-to-noise ratio of $10^{2}$. The reconstruction is indicated by a full curve whereas the broken curve represents the reconstruction obtained with a bounded support $[0.2,1.8]$. 


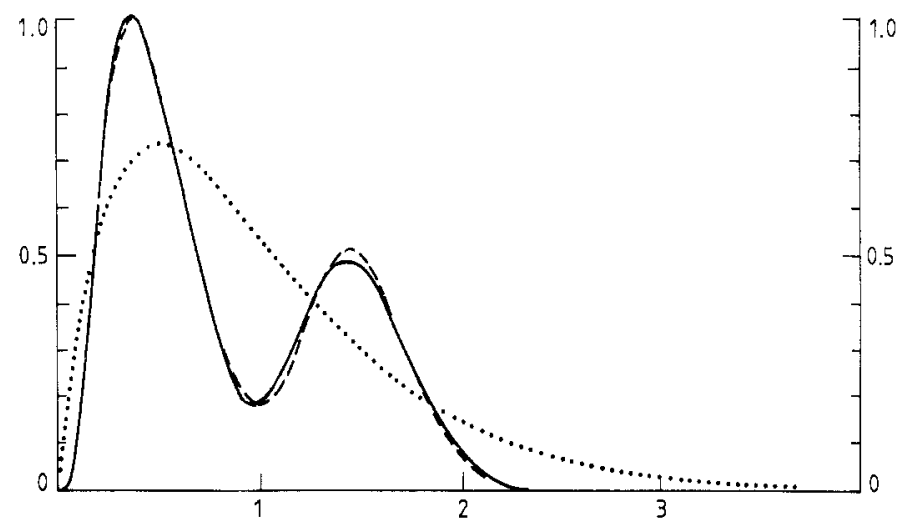

Figure 5. Reconstruction (full curve) of a sum of two gamma distributions (broken curve), using the profile (3.6) with $\beta=2$ (dotted curve) and for a signal-to-noise ratio of $10^{3}$. The data set was given by (2.18) with $N=31, x_{1}=0.001, x_{2}=15$.

singular functions $u_{N, k}$ and the data vector in the singular vectors $v_{N, k}$. The following expansion follows then from (2.4):

$$
f(r)=\sum_{k=0}^{K} \frac{g_{N, k}}{\alpha_{N, k}} u_{N, k}(r)
$$

where

$$
g_{N, k}=\sum_{n=1}^{N} w_{n} g\left(\xi_{n}\right) v_{N, k}\left(\xi_{n}\right)
$$

The number of terms used in (4.3) is in general smaller than $N$ and has to be chosen as a function of the signal-to-noise ratio $E / \varepsilon$. Stable solutions in the presence of noise are obtained by summing over all singular values larger than $\varepsilon / E$.

A good probe for estimating the achievable resolution is provided by reconstructions of delta distributions. We give an example in figure 4 for which the original distribution was a sum of two delta functions centred respectively at 0.725 and 1.275 . The data were sampled at the same set of points and with the same profile function as in figure 3 . The reconstruction takes into account all singular values larger than $10^{-2}$. We also present a reconstruction of the same object from the same data but using no profile and assuming that the support of the object is the interval $[0.2,1.8]$. We see in this figure an example of the undesirable edge effects which are inherent to the case of bounded support.

Finally, in figure 5, we give the reconstruction of a sum of two gamma distributions of the form $f(r)=C r^{\alpha} \exp (-\beta r)$, where the parameters $C, \alpha$ and $\beta$ have been adjusted in order that the first distribution has mean value 0.5 , standard deviation 0.25 and maximum value 1 whereas the second distribution has mean value 1.5 , standard deviation 0.25 and maximum value 0.5 . We started from simulated data sampled at $N=31$ points again forming a geometric progression with $x_{1}=0.001, x_{2}=15$ and $\Delta=1.37785$. We used a profile function of the form (3.6) with $\beta=2$ and we have taken into account in the sum (4.3) all singular values larger than $10^{-3}$. The reconstruction is in very good agreement with the original object. 


\section{Acknowledgments}

C De Mol is 'Chercheur qualifié' of the Belgian National Fund for Scientific Research. This work has also been partially supported by the NATO Grant No $463 / 84$. The authors are grateful to Dr Paul Walters for stimulating their interest in this problem.

\section{References}

[1] Pike E R, McWhirter J, Bertero M and De Mol C 1984 Proc. IEE 131 660-7

[2] Bertero M, De Mol C and Pike E R 1985 Inverse Problems 1 301-30

[3] Twomey S 1965 J. Franklin Inst. 279 95-109

[4] Twomey S 1974 Appl. Opt. 13 942-5

[5] Capps C D, Henning R L and Hess G M 1982 Appl. Opt. $213581-7$

[6] Bertero M, Brianzi P, Parker P and Pike E R 1984 Opt. Acta. 31 181-201

[7] Bertero M, Brianzi P and Pike E R 1985 Proc. R. Soc. A 398 23-44

[8] Bertero M, Boccacci P and Pike E R 1985 Inverse Problems 1 111-26

[9] Cummins H Z and Pike E R (ed.) 1974 Photon Correlation and Light Beating Spectroscopy (New York: Plenum)

[10] Bertero M and Pike E R 1983 Opt. Acta. $301043-9$

[11] Van de Hulst 1957 Light Scattering by Small Particles (New York: Wiley)

[12] Shifrin K S and Perelman A Ya 1963 Opt. Spectrosc. 15 285-9

[13] Walters P T 1980 Appl. Opt. $192353-65$

[14] McWhirter J G and Pike E R 1978 J. Phys. A: Math. Gen. 11 1729-45

[15] Bertero M, De Mol C, Pike E R and Walker J G 1984 Opt. Acta. 31 923-46

[16] Bertero M, Brianzi P and Pike E R 1985 Inverse Problems 1 1-15

[17] Byrne C L and Fitzgerald R M 1982 SIAM J. Appl. Maths 42 933-40

[18] Byrne C L and Fitzgerald R M 1984 SIAM J. Appl. Maths 44 425-42

[19] Hille E and Tamarkin T D 1931 Acta. Math. 57 1-76

[20] Riesz F and S Nagy B 1955 Functional Analysis (New York: Ungar)

[21] Erdelyi A 1953 Higher Transcendental Functions vol. 2 (New York: McGraw-Hill) 